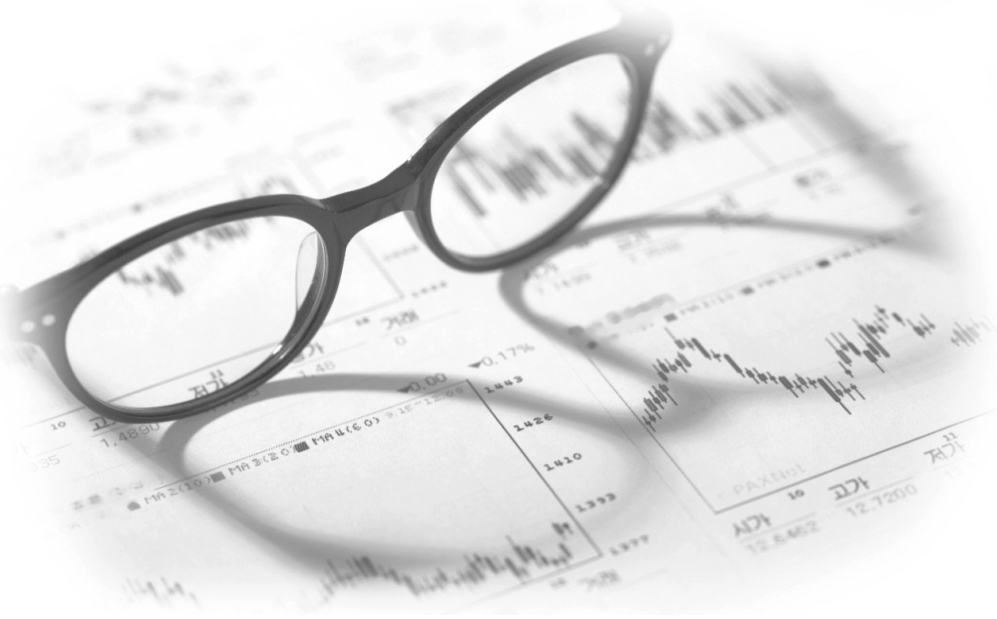

http://dx.doi.org/10.5045/kjh.2011.46.4.211

The Korean Journal of Hematology Volume 46 • Number 4 • December 2011 Perspective

\section{Lymphoma stem cells: A step toward a new therapeutic target}

\author{
Seok Jin Kim, M.D., Ph.D.
}

Division of Hematology-Oncology, Department of Medicine, Samsung Medical Center, Sungkyunkwan University School of Medicine, Seoul, Korea

\section{INTRODUCTION}

Since lymphoma is a chemosensitive neoplasm, a complete response can be achieved by systemic chemotherapy, even in the metastatic setting. Even so, relapse occurs in a substantial number of patients with an apparently complete clinical response. It is therefore possible that a subset of lymphoma cells that remain viable during treatment causes the relapse. The cancer stem cell hypothesis postulates the presence of this specific cell population in cancers. It suggests that cancers are derived from cancer stem cells, which are a particular subset of cancer cells with stem-like properties, including self-renewal [1]. Since the existence of cancer stem cells was demonstrated in acute myeloid leukemia [2], various surface markers have been identified to isolate cancer stem cells from such solid cancers as breast, brain, and colon. Although a recent publication suggested the probability of lymphoma stem cells in non-Hodgkin lymphoma [3], it did not offer sufficient data to support the presence of cancer stem cells as an origin of lymphoma. However, given the potential role of cancer stem cells in the pathogenesis of cancers, the possibility of lymphoma stem cells deserves attention, for it could improve our understanding of lymphoma biology and lead to the development of a new therapeutic target for lymphomas.

\section{STEM CELLS IN HODGKIN LYMPHOMA}

A small population of malignant cells, less than $1 \%$ of the total cells in this disease entity, is the hallmark of Hodgkin lymphoma. These malignant cells bear little phenotypic resemblance to normal $\mathrm{B}$ cells, including dim and variable CD20 expression. This unique nature of Hodgkin lymphoma has suggested the existence of lymphoma stem cells. A previous study has demonstrated the presence of a small population of CD20-positive B cells in 2 Hodgkin lymphoma cell lines, L428 and KM-H2 [4]. These cells showed high expression of aldehyde dehydrogenase $(\mathrm{ALDH})$, a marker for cells having stem-like properties as well as the memory B-cell antigen CD27. The growth of Reed-Sternberg cells from an in vitro culture of these $B$ cells provides evidence for their stem cell-like property. These clonal B cells with $\mathrm{CD} 27^{+}$, and elevated ALDH were also detected in the peripheral blood of patients with classic Hodgkin lymphoma, although their clinical significance in Hodgkin lymphoma was not determined in this study. These results therefore strengthen evidence for the existence of lymphoma stem cells in Hodgkin lymphoma.

\section{STEM CELLS IN NON-HODGKIN LYMPHOMA}

Non-Hodgkin lymphoma refers to a group of heterogeneous disorders, including $\mathrm{B}$ cell and T/NK cell lymphomas. It is classified into various subtypes depending on the morphologic, immunophenotypic, and clinical characteristics. These subtypes of non-Hodgkin lymphoma are associated with the specific developmental stages of the counterpart normal immune cells. For instance, errors in any of the immunoglobulin gene rearrangements during the developmental process of normal B cells may result in aberrant chromosomal translocations involving immunoglobulin heavy chain genes, which are characteristic of different

This is an Open Access article distributed under the terms of the Creative Commons Attribution Non-Commercial License (http://creativecommons.org/licenses/by-nc/3.0) which permits unrestricted non-commercial use, distribution, and reproduction in any medium, provided the original work is properly cited. 
subtypes of B-cell non-Hodgkin lymphoma [5]. This implies that a subset of cells with a genetic mistake occurring during their development might be an origin of lymphoma, and suggests that they could play a role as lymphoma stem cells. However, the concept of cancer stem cells would not necessarily imply that the transformation of normal stem cells constitutes the initiating step of carcinogenesis. If normal mature cells could acquire some properties of normal stem cells after their transformation into premalignant cells, they would re-express properties of stem cells, and then initiate cancers. Likewise, premalignant lymphoma cells might need additional genetic changes associated with stemness to become lymphoma stem cells. For example, the first chromosomal translocations could occur in mature lymphocytes within the germinal center, and these would need to accumulate secondary reprogramming mutations in order to develop germinal center-derived lymphomas.

\section{SIDE POPULATION CELLS IN HODGKIN AND NON- HODGKIN LYMPHOMAS}

Side population (SP) analysis has been used to exploit the presence of cancer stem cells for cancers that have no surface marker for stem cell isolation. This analysis is based on the peculiar characteristic of normal stem cells that allows them to protect themselves from cytotoxic agents via high expression of ATP-binding cassette (ABC) transporters. Thus, the SP is a sub-population of cells that extrude the cell-permeable DNA-specific dye, Hoechst 33342, through an ABC transporter. The SP was recognized as a stem cell population, distinguishable from other such populations [6]. Such a distinguishing property supports that notion that cancer stem cells are associated with a resistance to chemotherapy and posttreatment relapse. Previous studies in several human and murine cancer cell lines have demonstrated that SP cells could be characterized as cancer stem cells. In Hodgkin lymphoma, the possibility of lymphoma stem cell population and the association of this population with resistance to chemotherapy have been reported. Thus, Hodgkin lymphoma cell lines contain SP cells, which are enriched for distinct small mononuclear cells and generate larger cells with Reed-Sternberg cell-like morphology [7]. In addition, the SP cells in Hodgkin lymphoma cell lines show increased resistance to gemcitabine, a drug commonly used to treat refractory Hodgkin lymphoma [8]. A study with a murine mantle cell lymphoma model also proposed that SP cells contain tumor-initiating cells responsible for lymphoma maintenance and dissemination [9]. Our own group has isolated SP cells from human B-cell non-Hodgkin lymphoma cell lines and from primary cells derived from refractory B-cell non-Hodgkin lymphoma patients (Fig. 1). In our study, the characteristics and gene expression profiles of SP cells were different from those of the main population within the same cell lines. All these results using SP analysis suggest the probability of a specific subset of lymphoma cells in Hodgkin and non-Hodgkin lymphoma that might be enriched with lymphoma stem cells.

\section{CONCLUSIONS}

The concept of cancer stem cells is changing our understanding of cancer biology. Thus, the identification of cancer stem cells may have profound effects on cancer diagnostics and therapeutics, changing the paradigm of management. The existence of lymphoma stem cells remains uncertain. However, if specific markers-such as the CD45+CD19cells proposed in a recent study as stem-like cells in human mantle cell lymphoma [10]-are identified, evidence in support of the existence of lymphoma stem cells might be

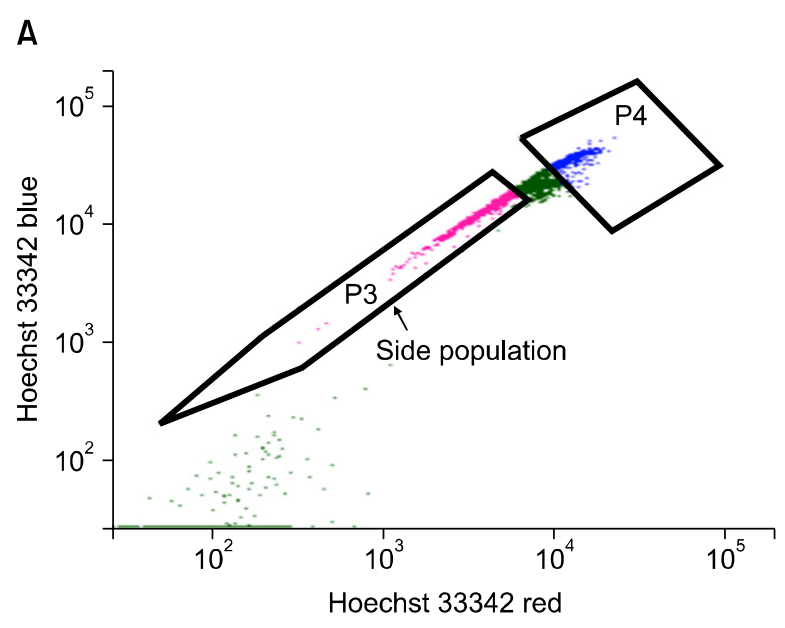

B

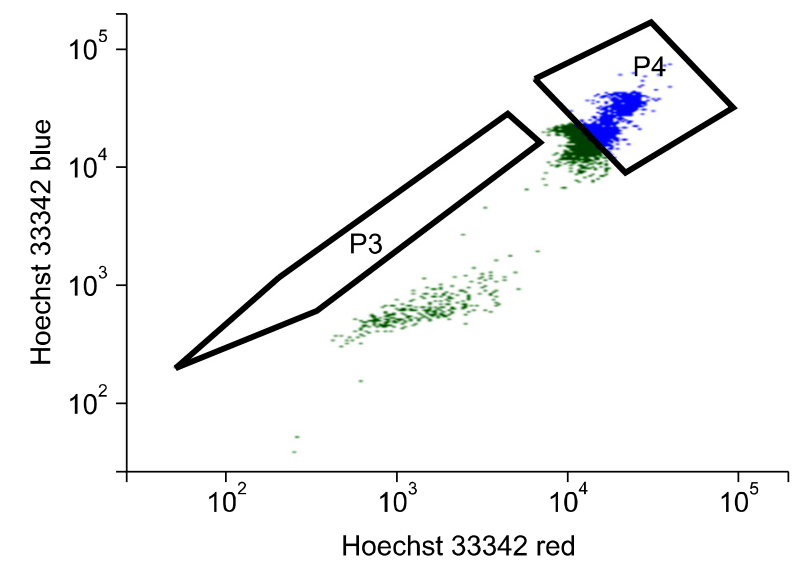

Fig. 1. Primary cells from malignant ascites of a refractory B-cell non-Hodgkin lymphoma patient are sorted as a side population (P3) and a non-side population (P4) using flow cytometry (A). The side population (P3) in the tail region of the dot plot is reduced when the cells are incubated with verapamil (B). 
accumulated in the future. Thus, further studies to characterize lymphoma stem cells in lymphoma could contribute to the development of a new therapeutic target for lymphoma.

*This work was supported by the Basic Science Research Program through the National Research Foundation of Korea (NRF), funded by the Ministry of Education, Science and Technology (2009-0075642).

\section{REFERENCES}

1. Reya T, Morrison SJ, Clarke MF, Weissman IL. Stem cells, cancer, and cancer stem cells. Nature 2001;414:105-11.

2. Warner JK, Wang JC, Hope KJ, Jin L, Dick JE. Concepts of human leukemic development. Oncogene 2004;23:7164-77.

3. Martinez-Climent JA, Fontan L, Gascoyne RD, Siebert R, Prosper F. Lymphoma stem cells: enough evidence to support their existence? Haematologica 2010;95:293-302.

4. Jones RJ, Gocke CD, Kasamon YL, et al. Circulating clonotypic B cells in classic Hodgkin lymphoma. Blood 2009;113:5920-6.
5. Willis TG, Dyer MJ. The role of immunoglobulin translocations in the pathogenesis of B-cell malignancies. Blood 2000;96: 808-22.

6. Goodell MA, Brose K, Paradis G, Conner AS, Mulligan RC. Isolation and functional properties of murine hematopoietic stem cells that are replicating in vivo. J Exp Med 1996;183: 1797-806.

7. Nakashima M, Ishii $Y$, Watanabe M, et al. The side population, as a precursor of Hodgkin and Reed-Sternberg cells and a target for nuclear factor- $\kappa \mathrm{B}$ inhibitors in Hodgkin's lymphoma. Cancer Sci 2010;101:2490-6.

8. Shafer JA, Cruz CR, Leen AM, et al. Antigen-specific cytotoxic T lymphocytes can target chemoresistant side-population tumor cells in Hodgkin lymphoma. Leuk Lymphoma 2010;51:870-80.

9. Vega F, Davuluri Y, Cho-Vega JH, et al. Side population of a murine mantle cell lymphoma model contains tumour-initiating cells responsible for lymphoma maintenance and dissemination. J Cell Mol Med 2010;14:1532-45.

10. Jung HJ, Chen Z, McCarty N. Stem-like tumor cells confer drug resistant properties to mantle cell lymphoma. Leuk Lymphoma 2011;52:1066-79. 